\title{
Tārah 5@: modelo de intervención para la atención de la violencia contra las mujeres, las adolescentes $y$ las niñas desde un enfoque de seguridad y procuración de justicia
}

\section{Tärah 5@: intervention model for violence prevention against adult, teenage, and infant women, from a security and law enforcement perspective}

\author{
Carolina Rea Gómez \\ Universitat de Girona, España \\ caroreag@hotmail.com \\ Alfonso J. Motta Allen \\ Universidad de Quintana Roo, México \\ alfonso.motta@uqroo.edu.mx \\ https://doi.org/10.36105/iut.2021n34.07
}

Recibido: $21 / 11 / 2021$

Aceptado: 20/12/2021

\section{RESUMEN}

Tārah 5 es un modelo empírico en desarrollo para combatir principalmente y de manera urgente, la violencia contra las mujeres, las adolescentes y las niñas interrumpiendo el continuo de la violencia para evitar más feminicidios. Ha sido ideado a partir del conocimiento y la experiencia sobre los sistemas existentes de seguridad y procuración de justicia en México, y sobre esta base se enfoca en la necesidad de identificar esfuerzos y estructuras existentes en una localidad para lograr que funcionen como un todo. La naturaleza de la violencia contra las

(c) Derechos Reservados. Carolina Rea Gómez y Alfonso J. Motta Allen, 2019, Registro ante INDAUTOR, 2019.

(c) All rights reserved. Carolina Rea Gómez and Alfonso J. Motta Allen, 2019, Registration with INDAUTOR, 2019. 
mujeres, las adolescentes y las niñas requiere que cada caso se atienda de manera individual y de forma articulada. La dimensión del problema demanda explorar todas las formas y enfoques posibles para cambiar positivamente la realidad de millones de mujeres, adolescentes y niñas que hoy son víctimas de algún nivel de violencia por el solo hecho de ser mujeres; y las cifras de mujeres asesinadas exige respuestas urgentes. La que aquí se presenta es una propuesta que los autores hacen desde la conjunción de sus propias plataformas de especialización.

Palabras clave:

violencia de género, feminicidio, impartición de justicia.

Abstract:

Tarah 5 is a developing empirical model to counter in an urgent manner violence against adult, teenage and infant women, to interrupt the violence continuum to prevent more femicides. It has been devised derived from the knowledge and experience on the current security systems and law enforcement in Mexico. It focuses on the need to identify actions and existing structures in a location, for them to work as a whole. The nature of the violence against women of all ages requires that each case is attended individually and in an articulate matter. The dimension of this problem demands to explore all forms and possible approaches to it in order to positively affect the reality of millions of women that are currently victims of any type of violence, only because of the fact that they are women. The statistics on murdered women demand urgent answers. The one provides here is a proposal that the authors contrived from the combination of their areas of expertise.

Keywords:

Gender-based violence, femicide, administration of justice.

Nota introductoria

La ONU reporta que la violencia contra las mujeres, las adolescentes y las niñas en América Latina y el Caribe, en su expresión más violenta, el feminicidio, constituye una amenaza significativa. De los 25 países con los índices de femicidio más altos del mundo, 14 se encuentran en América Latina y el Caribe. Según la CEPAL, 12 mujeres mueren en promedio cada día en la región por el solo hecho de ser mujeres. En América Central, dos de cada tres asesinatos de mujeres están relacionados con el género. Algunos de estos países tienen las tasas de homicidio más altas del mundo y en ellos el número de muertes violentas es cuatro veces mayor para las mujeres que para los hombres. Estudios realizados en Brasil estiman que una pareja íntima mata a una mujer cada seis horas. El estudio global más reciente sobre el homicidio muestra que una pareja íntima o un miembro de 
la familia es responsable de la muerte de una de cada dos mujeres, contrastando con las muertes de hombres que es una de cada 20 para este caso. Estos asesinatos a menudo no son incidentes aislados, sino que ocurren dentro de un continuo de inseguridad ciudadana. ${ }^{1}$

\section{¿A qué nos referimos cuando hablamos de la violencia contra las mujeres, las ado- lescentes y las niñas?}

La violencia de género es la violencia dirigida contra una persona con base en su identidad de género, expresión de género o percepción de género. En ese sentido, tanto las mujeres como los hombres pueden ser víctimas de violencia de género, pero la mayoría son mujeres, adolescentes y niñas. Las últimas estimaciones de la Organización de las Naciones Unidas indican que aproximadamente un tercio de las mujeres de todo el mundo ha sufrido violencia física o sexual alguna vez en su vida y dos de cada tres víctimas de homicidios relacionados con la pareja o la familia son mujeres. ${ }^{2}$

Eliminar la violencia contra las mujeres, las adolescentes y las niñas es imprescindible para garantizarle a ellas el ejercicio pleno del derecho fundamental a la vida, la libertad, la seguridad, la dignidad, la igualdad frente a los hombres, la no discriminación y la integridad física y mental. ${ }^{3}$

La violencia contra las mujeres, las adolescentes y las niñas puede provenir de una variedad de factores, pero feministas y académicos reconocen en gran medida que las causas subyacentes de esta violencia provienen de las estructuras de poder patriarcales y de las culturas en las que los hombres tienen el control, en particular sobre la vida sexual y reproductiva de las mujeres, desde el aparato del Estado hasta la familia. ${ }^{4}$

En ese sentido las sociedades mantienen, a través de sus estructuras, costumbres y valores, distintos niveles de violencia institucionalizada o "normalizada" y de discriminación que sitúa a las mujeres, entre otros grupos de personas, por debajo de sus potenciales de autorrealización física y mental. ${ }^{5}$

Por esa razón la sola atención de los casos de violencia física es insuficiente para llegar a las causas y por ello para atender de manera efectiva el problema de la violencia contra las mujeres, las adolescentes y las niñas se requiere un enfoque amplio, es decir, concibiendo el problema en todos sus aspectos y

\footnotetext{
1 Spotlight Initiative to eliminate violence against women and girls, Terms of Reference 2017-2023, UN \& EU, 2017, https://www.spotlightinitiative.org/

2 United Nations Sustainable Development Goal 5 Achieve gender equality and empower all women and girls. https://sustainabledevelopment.un.org/sdg5

3 Declaración sobre la eliminación de la violencia contra la mujer, proclamada por la Asamblea General en su resolución 48/104 de 20 de diciembre de 1993, https://www.ohchr.org/EN/ProfessionalInterest/Pages/ ViolenceAgainstWomen.aspx

4 Gould, Laurie A., Exploring the Relationship Between Gender Violence and State Failure: A CrossNational Comparison, Georgia Southern University, Statesboro, USA, Violence Against Women 2016, vol. 22(11) 1343-1370, p. 1344, sagepub.com/journalsPermissions.nav, https://doi.org/10.1177/1077801215624790

5 Galtung, Johan, Violence, Peace, and Peace Research, p. 169, Journal of Peace Research, vol. 6, núm. 3 (1969), pp. 167-191, Published by: Sage Publications, Ltd.Stable, http://www.jstor.org/stable/422690.
} 
considerándolo como un todo para lograr con ello hacer "visibles" aquellos aspectos de la violencia que "normalmente" son invisibles, e incluirlos, junto con los aspectos "visibles" de la violencia, en la solución al problema. Sin embargo, al entender la complejidad del problema y la dificultad para modificar estructuras sociales tan arraigadas, la intención de los autores es encausar una intervención inmediata que atienda las manifestaciones de violencia más graves y extremas.

\section{Significado del nombre TĀRAH 5}

Tārah 5 es un nombre estilizado tomado de Tara, concepto que tiene un campo semántico muy amplio; su nombre en sanscrito significa "protectora", es también un mantra que encarna muchas de las cualidades del principio femenino y que alude a la capacidad de acción y de superación de obstáculos. Esta palabra refiere a la liberación, a las acciones decisivas y dinámicas y entre los budistas representa una especie de revolución feminista ecuánime.

Aunque la palabra tara en castellano tiene una connotación negativa (la RAE la define como: "Defecto físico o psíquico, por lo común importante y de carácter hereditario"), ${ }^{6}$ los autores decidimos mantener el nombre porque es precisamente el objeto del modelo y el motor del esfuerzo: combatir a la violencia contra las mujeres como un defecto estructural y hereditario de la sociedad. La fusión de ambas definiciones, creemos, nos ofrece esa dualidad de reto y esperanza que el modelo necesita para alcanzar sus objetivos en términos de prevención y de protección.

$\mathrm{Y}$ el número 5 es porque buscamos que este modelo contribuya al logro del Objetivo 5 de Desarrollo Sostenible 2030, en particular los objetivos 5.1 y 5.b que se refieren, respectivamente, a 1) erradicar todas las formas de discriminación contra las mujeres, los adolescentes y las niñas en todo el mundo; y 2) fomentar el uso de tecnologías facilitadoras, en particular las tecnologías de la información y las comunicaciones, para promover el empoderamiento de la mujer.

\section{El concepto TĀRAH 5}

La violencia contra la mujer es un problema prevalente con importantes consecuencias para la salud física y mental en todo el mundo, y si bien una política pública sólida depende de mediciones precisas, la cuantificación de los aspectos invisibles de la violencia contra las mujeres, las adolescentes y las niñas sigue siendo uno de los mayores retos sociales. Tārah 5 dimensiona el problema de la violencia contra las mujeres, las adolescentes y las niñas a partir del marco conceptual propuesto por Johan Galtung, que, desde la perspectiva de los estudios de paz, entrelaza tres aspectos que describen la relación entre lo invisible y lo visible de la violencia, argumentando que la violencia es cultural, estructural y directa. ${ }^{7}$ Esta base conceptual la modifica Sarah Henkeman para acomodarla a un

6 Diccionario de la lengua española, Real Academia Española, edición Tricentenario, actualización 2020, https:// dle.rae.es/tara?m=form.

7 Kirthi Jayakumar, Redrawing the Galtung Triangle - Finding Place for Healing Trauma in Peace Work, 17 Jun 2019, https://www.transcend.org/tms/2019/06/redrawing-the-galtung-triangle-finding-place-for-healingtrauma-in-peace-work/ 
contexto de inequidad "normalizada" que busca liberarse de una realidad de trauma histórico. ${ }^{8}$

Lamentablemente la violencia contra las mujeres, las adolescentes y las niñas se dimensiona comúnmente a partir de su componente visible, dejando fuera los componentes invisibles o "invisibilizados" por las estructuras de poder, las culturas y la violencia psicológica relacionada, por lo que la cuantificación de la violencia contra las mujeres, las adolescentes y las niñas sigue siendo uno de los mayores retos sociales.

Con ello en mente y con la finalidad de dimensionar mejor el problema y definir acciones concretas, Tārah 5 se enfoca en que se atiendan de manera urgente los aspectos de violencia física, así como los estructurales, culturales y psicológicos, visibles e invisibles, y de ahí su perspectiva amplia de análisis y de acción coordinada como un todo. Esto significa ver los aspectos del problema no de forma aislada y actuar para "visibilizar" los que se encuentren ocultos, e interrumpir patrones de violencia "normalizada" y secuencias de violencia física y psicológica.

Con la finalidad de abarcar de manera práctica los aspectos más significativos para generar cambios en las estructuras y las culturas de la violencia, Tārah 5 agrega a la base teórica propuesta por Galtung y Henkeman, las consideraciones de tres de los enfoques dominantes que vinculan el género con la violencia: el continuo de la violencia, el análisis interseccional del poder, y el modelo socio ecológico.

\section{Continuo de la violencia}

Este enfoque sugiere que existe una secuencia de hechos violentos de género que se extiende desde lo individual hasta lo nacional e internacional; desde el hogar hasta las escenas del crimen o hasta los campos de batalla. ${ }^{9}$ Este concepto identifica vínculos significativos entre la violencia contra la mujer en los espacios privados como el hogar, y en los espacios públicos, tanto en contextos seguros como en contextos delictivos o de conflicto. El enfoque enfatiza un flujo ininterrumpido o secuencia de eventos violentos y vincula tipos de opresión, violencia y escalas de fuerza (agresiones verbales y psicológicas, agresiones físicas, privaciones de la vida) de manera individual y de grupo (dos personas en una pelea, peleas entre grupos o guerras entre naciones) que resultan en varias categorías de violencia de género. ${ }^{10}$

Si bien este enfoque tiene el inconveniente que no logra entrelazar los hechos violentos con sus causas, particularmente con las características no lineales y las

\footnotetext{
8 Henkeman, Sarah et al, Transdisciplinary project for social justice, Open Guide to a Deeper, Wider and Longer Analysis of Violence, 2016, https://www.researchgate.net/publication/312587585_Open_Guide_to_a_Deeper_ Wider_and_Longer'_analysis_of_violence

9 Gray, Harriet and Cockburn, Cynthia, "Anti-Militarism: Political and Gender Dynamics of Peace Movements, International”, Feminist Journal of Politics, 16:1, 168-170, https://doi.org/10.1080/14616742.2013.8 $76293,2014$.

10

Idem.
} 
reproductoras de la violencia, el modelo Tārah 5 lo encuentra adecuado para enfocar los esfuerzos de prevención y de protección, particularmente de la integridad física de las mujeres, adolescentes y niñas, y ayuda a organizar dichos esfuerzos de acuerdo con su especialización y a priorizarlos para aumentar su capacidad preventiva, permitiendo con ello que se pueda actuar en las etapas tempranas de la violencia. En otras palabras, el modelo Tārah 5 identifica los hechos violentos más comunes dentro del continuo de violencia y articula la acción necesaria para interrumpir dicho patrón y evitar así que la violencia escale hasta el feminicidio.

\section{Análisis interseccional de poder}

El "análisis interseccional del poder" revela la manera en que las diferentes dimensiones del poder, como el capitalismo, la dominación etno-nacional y el patriarcado, se refuerzan mutuamente. Las intersecciones de varias divisiones sociales, como la clase, el género, la etnia y la raza, refuerzan las formas ya existentes de discriminación y opresión, lo que da lugar no sólo a una representación más pobre de las mujeres pertenecientes a minorías, sino también a una mayor opresión. ${ }^{11}$ Este modelo interseccional, sin embargo, no alcanza a explicar la violencia que resulta cuando otras manifestaciones de poder como el racismo, el sexismo y el nacionalismo se entrelazan estrechamente.

A partir de su capacidad analítica, Tārah 5 se apoya en el análisis interseccional del poder para sugerir la elaboración de expedientes individuales de las personas hacia las que va dirigido, distinguiendo los niveles de riesgo de violencia a los que están expuestas, de manera real y potencial, a partir de distinguir en cada una de estas personas su pertenencia o relación con dichas divisiones de poder y activando las bases argumentativas para la generación de acuerdos entre los actores participantes en el modelo; de ahí la importancia de su implementación local.

\section{Modelo socio ecológico}

El modelo socio ecológico explica que un marco integrado de violencia debe identificar y combinar cuatro niveles diferentes: estructural, institucional, interpersonal o comunitario, e individual, ya que "ningún nivel causal por sí solo determina la violencia, sino que cuando cada uno se combina con una o más variables causales adicionales, puede dar lugar a una situación en la que se produce violencia". ${ }^{12}$

Este modelo logra entrelazar los roles de género a la vez que permite ver los tipos de abusos socioculturales, políticos y económicos perpetrados por individuos,

11 Hughes, Melany, "The intersection of gender and minority status in national legislatures: The minority women legislative index", Legislative Studies Quarterly, XXXVIII, 4, November 2013, 489, https://doi.org/10.1111/ 1sq. 12025.

12 Moser, Caroline, "The gendered continuum of violence and conflict: an operational framework", 2001. In: Caroline Moser and Fiona Clark (eds), Victims, Perpetrators or Actors? Gender, Armed Conflict and Political Violence. London: Zed Books. In: A holistic approach to violence: Women parliamentarians' understanding of violence against women and violence in the Kurdish issue in Turkey, p. 78, European Journal of women's Studies 2016, vol. 23(1) 76-92, 2014, sagepub.co.uk/journalsPermissions.nav https://doi.org/10.1177/1350506814554487 
grupos y gobiernos. Con ello el modelo socio ecológico explica por qué la violencia contra la mujer se normaliza a través de los roles de género y de las enseñanzas culturales y permanece en tanto las instituciones, normas y estructuras que la alimentan, no son transformadas o eliminadas. Al observar que hay una conexión directa entre "el daño por opresión" y "el daño físico", ${ }^{13}$ los académicos han argumentado que la violencia estructural en forma de pobreza, exclusión y humillación, por ejemplo, "inevitablemente se traduce en violencia íntima y doméstica". ${ }^{14}$

Para el modelo Tārah 5 el enfoque del modelo socio ecológico resulta de gran utilidad para evidenciar las relaciones causales a través del análisis individual de perfiles y niveles de riesgo y de manera general mediante la interposición de capas de ubicación geográfica de acciones y omisiones de sociedad y gobierno; posible en gran medida gracias a la capacidad de denuncia de Tārah 5. Para ello el modelo Tārah 5 requiere de la producción de mapas de violencia que muestren gráficamente las áreas de "coincidencia" geográfica, por ejemplo, donde se observe la intersección de áreas de la localidad donde existe el mayor número de hechos de violencia doméstica, con áreas que tienen los índices más altos de pobreza, menor número de centros de salud, y menor número de escuelas, entre otros. De esta manera se puede observar con claridad las áreas o zonas de la ciudad donde la sociedad y el gobierno locales ponen más y qué recursos, y donde menos.

\section{El tablero de acción TĀRAH 5}

Ideado como un modelo de seguridad y de procuración de justicia, una vez que ha dimensionado a detalle el problema de la violencia contra las mujeres, las adolescentes y las niñas y su naturaleza manifiesta visible e invisible, Tārah 5 identifica los esfuerzos locales existentes y sus correspondientes actores, los enlaza, comunica, refacciona y equipa con recursos adicionales, para articular toda esta acción como un todo, y lo hace de manera individual para cada persona usuaria, o sea, para cada persona que se busca proteger. Por decirlo de otra manera, Tārah 5 logra que todos los actores locales intervengan según su especialidad y de forma coordinada, en cada uno de los casos individuales de violencia, logrando articular masivamente y potenciar a niveles superiores todos los esfuerzos y recursos existentes en una localidad. El fin superior de Tārah 5 es lograr que a través de las capacidades que ofrece este modelo se interrumpa el continuo de la violencia en cualquiera de los puntos, desde los más invisibles hasta el más visible y extremo, evitando con ello los feminicidios.

A partir del modelo conceptual Galtung/Henkeman y de los enfoques dominantes que vinculan el género con la violencia, el modelo Tārah cuenta con un tablero de acción sobre el que se organizan y articulan tales actores y sus esfuerzos existentes. Consecuentemente, el modelo muestra gráficamente hacia qué aspectos

13 Cockburn, Cynthia, "The Continuum of Violence: A Gender Perspective on War and Peace", Wenona Giles, Sites of Violence: Gender and Conflict Zones, California Scholarship Online: March 2012, http://doi. org/10.1525/california/9780520230729.001.0001.

14 SChePer-Hughes, Nancy, Death Without Weeping: The Violence of Everyday Life in Brazil, University of California Press, 1992, p. 614. 
dicha localidad ha dirigido la acción y hacia cuales la inacción o la indiferencia en el combate a la violencia contra las mujeres, las adolescentes y las niñas.

El tablero se forma cruzando dos ejes capitales: el de la acción de la seguridad (protección y prevención), con el de la naturaleza de la violencia contra las mujeres, las adolescentes y las niñas (visible e invisible), creándose así cuatro campos o cuadrantes de acción: Q1, Q2, Q3, y Q4.

De esta forma se puede anotar en el tablero a todos los actores locales con los cuales se va a trabajar a través del modelo Tārah 5 de acuerdo con sus respectivas especialidades y capacidades. La colocación de los actores en los distintos cuadrantes corresponderá a la orientación de sus acciones y esfuerzos según corresponda a su nivel de prevención y protección y a lo visible o invisible que sea el o los aspectos de la violencia que atienden.

Cuando y donde no existan actores o capacidades para cubrir alguna de las especialidades necesarias para la interrupción del continuo de la violencia (sensibilización, empoderamiento, apoyo psicológico, etc.), el modelo Tārah 5 ofrece la posibilidad de incluir tales necesidades a partir del uso de la tecnología, como se explica más adelante.

La Ilustración 1 muestra el Tablero de acción Tārah 5 con ejemplos de los tipos y objetivos de intervención según la especialización requerida. El continuo de la violencia se representa por una línea dinámica que une hechos violentos hipotéticos según sus características de visibilidad y necesidad de acciones de prevención-protección según corresponda su atención.

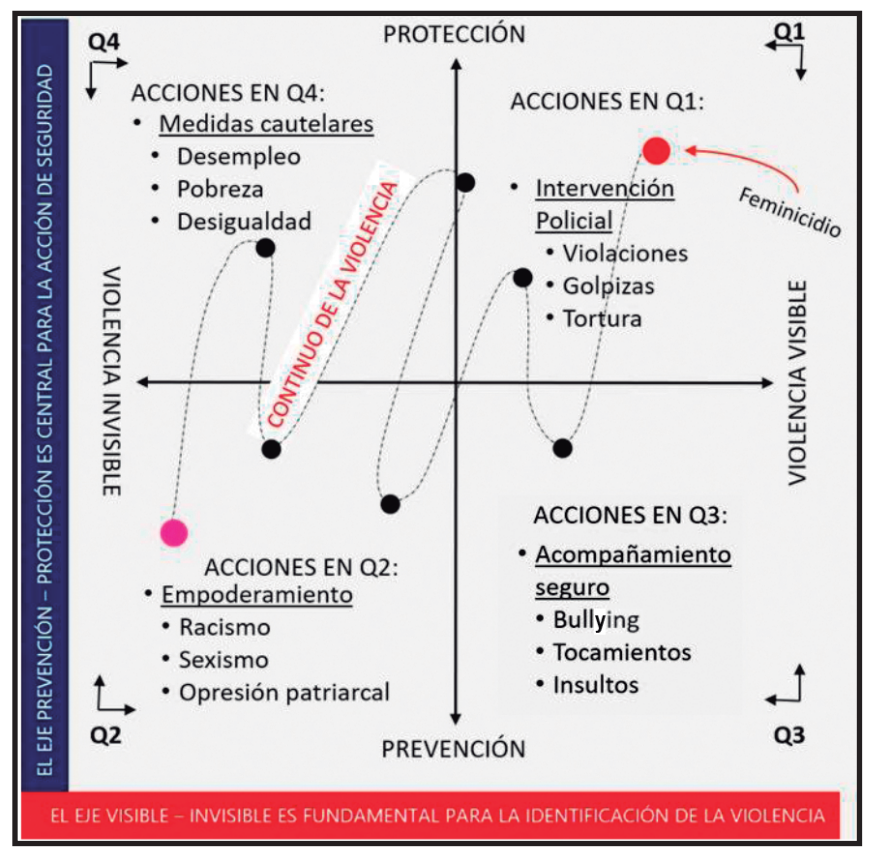

Ilustración 1. Tablero de Acción Tārah 5. Fuente: elaboración propia. 
Puede observarse en la representación gráfica del tablero que la parte más visible de la violencia se encuentra ubicada en Q1 y ahí se concentran los mayores esfuerzos de protección, referidos como acciones de intervención afirmativa para proteger o evitar daños a la integridad física de las personas, en forma de golpizas, amenazas de muerte, violaciones sexuales y muerte; los actores fundamentales para la acción en Q1 serán los que se encuentran en las estructuras estatales de la seguridad pública, siendo la policía el principal actor en este cuadrante, la que se conecta a Tārah 5 a través de sus centros de control operativo.

\section{Cuadrante de Acción Q2}

Diagonalmente opuesto a Q1 se encuentra Q2 y en él se ubican los ámbitos de mayor necesidad de sensibilización y empoderamiento y por ende de mayor capacidad de prevención. En Q2 los esfuerzos de sensibilización y empoderamiento responsable van dirigidos a evitar las acciones violentas generalmente invisibles, como chantajes, celos, menosprecio y control sobre mujeres, adolescentes y niñas. A través de acciones dirigidas en educación, concientización, apoyo emocional e intervención temprana, Tārah 5 busca interrumpir el continuo de la violencia y evitar con ello que los patrones de violencia deriven en acciones de violencia física y/o la muerte. En este cuadrante, los actores emblemáticos son las organizaciones internacionales o de la sociedad civil que cuentan con capacidades de apoyo emocional o psicológico, tales como líneas telefónicas de asistencia (hotlines).

\section{Cuadrante de Acción Q3}

Q3 incluye los ámbitos donde se dan las acciones de mayor violencia visible pero que requieren niveles relativamente más bajos de protección física. En este cuadrante las acciones son preventivas y van encaminadas a evitar que las personas usuarias sean víctimas de violencia física a través de un acompañamiento seguro en sus traslados en su vida cotidiana y en espacios públicos y privados.

\section{Cuadrante de Acción Q4}

El cuadrante Q4 incluye los ámbitos donde se requiere mayor protección contra hechos no siempre visibles y que requieren atención por su relación directa con hechos de violencia psicológica y estructural. Por la importancia y consideraciones prácticas de seguridad y procuración de justicia se han acomodado en este cuadrante las acciones de protección contra la "violencia judicializada", que se refiere a los casos en que la autoridad ministerial y/o judicial ya ha tomado conocimiento de los hechos. Los actores principales en este cuadrante son aquellos que se relacionan con el historial de abusos de las personas usuarias y su necesidad particular de protección contra los perpetradores de dichos abusos. 
Existen numerosos esfuerzos en el mundo orientados a combatir la violencia contra las mujeres, las adolescentes y las niñas desde distintos enfoques, pero hay muchos menos trabajando de manera orquestada en acciones concretas. Tārah 5 busca hacer realidad la posibilidad de que las comunidades locales trabajen de manera coordinada y efectiva en la solución de un problema que afecta directamente a la mitad de la población y que tiene implicaciones directas en toda la sociedad.

Por la naturaleza de la violencia contra las mujeres, las adolescentes y las niñas, su atención requiere de formas creativas de organización y coordinación, y de toma de decisiones de los diversos actores; demanda una participación comprometida y responsable. Con esto en mente y bajo el principio de acción no violenta, el modelo Tārah 5 requiere de una estructura de participación local que logre articular acciones concretas, orquestadas y comprometidas de sociedad y gobierno.

Tārah 5 propone un esquema de participación democrática responsable y sustentable que permita su implementación y operación y que haga coincidir los intereses comunes de los participantes con el fin último del conjunto, que es evitar los feminicidios a partir de la erradicación de toda forma de discriminación contra mujeres, adolescentes y niñas, y fomentando el uso de tecnologías facilitadoras, en particular las de la información y las comunicaciones para promover el empoderamiento de la mujer (Objetivo 5 de Desarrollo Sustentable 2030, metas 5.1 y $5 . b)$.

El esquema propuesto es uno de participación comprometida (engagement) horizontalmente estructurado, de manera que la sociedad dé forma a las opciones políticas y que el gobierno participe al mismo nivel de capacidad de decisión. De esta forma se "visibiliza" lo invisible de la violencia y permite a los actores locales existentes, de sociedad y gobierno, contribuir a los objetivos citados a partir de sus propias capacidades y especialidades de atención de la violencia contra las mujeres, las adolescentes y las niñas, trabajando de manera coordinada y balanceada en su conjunto, a lograr el mayor impacto positivo en el problema a nivel local; y permite de manera colegiada la toma de decisiones sobre el modelo Tārah 5 que impacten colectivamente a la comunidad. En otras palabras, se trata de crear una estructura de orden y transparencia para el aprovechamiento legítimo y responsable de, y alrededor de, el modelo Tārah 5.

\section{El componente tecnológico de TĀRAH 5 (app)}

Para lograr llegar a todo el universo de mujeres, adolescentes y niñas que habitan una localidad, sea esta una población pequeña o una gran ciudad, el modelo necesariamente considera el uso de la tecnología de la información, la comunicación y conocimiento profundo o inteligencia artificial. Por ello el modelo Tārah 5 propone la utilización de una aplicación o "App" para teléfono celular con una interfase para hacer posible la participación interactiva de los actores 
locales (Ilustración 2). El uso de la tecnología debe correr sobre los ejes de la confidencialidad y la seguridad de la información, negación de su uso como herramienta de control para las mujeres que se busca proteger, no revictimización, mínima discriminación, aprovechamiento del conocimiento específico para suplir las carencias locales, visibilización de acciones y conductas violentas, máxima protección y fiscalización de respuestas de sociedad y gobierno.

Se propone que la $A p p$ del modelo se "nativa de la nube", lo que significa la posibilidad de que opere con capacidades de procesamiento y almacenamiento de datos en la nube y no en un centro de datos físico, con lo que se reduce la vulnerabilidad a la intrusión y la manipulación, aumenta la protección de la información y de los datos personales, disminuye la discriminación implícita por el uso de la tecnología que normalmente está disponible sólo para quienes pueden pagarla, y aumenta su capacidad de replicación global.

Que se apoye en conceptos que generen la máxima confianza para las personas usuarias y los actores involucrados, como por ejemplo "Zero Trust", concepto de seguridad centrado en la idea de que, en términos de acceso a un sistema informático, como lo es una $A p p$, no se debe confiar automáticamente en ninguna de las personas involucradas dentro o fuera del mismo y, en cambio, se debe verificar todo y a cualquier persona o máquina que intente conectarse a la $A p p$ antes de conceder el acceso. ${ }^{15}$

Para enlazar al universo de mujeres, adolescentes y niñas de una localidad, con los actores involucrados que trabajan en algún aspecto de la violencia, una App con las características arriba descritas sería capaz de hacerlo y llevar a cabo una serie de funciones si se le equipa con las capacidades de aprendizaje automático o machine learning para la realización de sus funciones, que de acuerdo con el modelo de intervención podría llevarlas a cabo a través de los siguientes componentes:

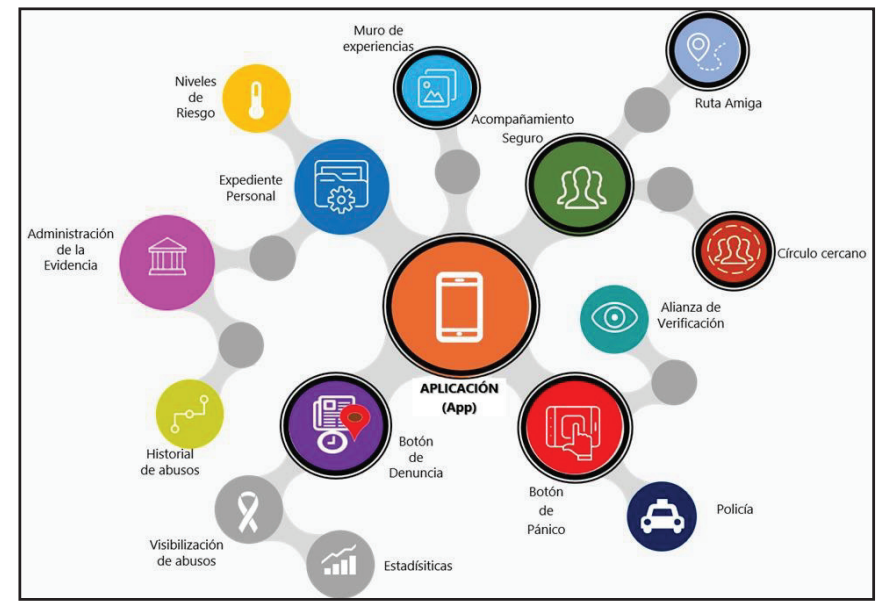

Ilustración 2. La App del Modelo Tārah 5. Fuente: elaboración propia.

15 KindervaG, John, Build Security into your network's DNA: The Zero Trust Network Architecture, Forrester Research, Inc., 2010, http://www.virtualstarmedia.com/downloads/Forrester_zero_trust_DNA.pdf 
Como su nombre lo sugiere, el Expediente personal sería un registro generado por la aplicación de manera individual para cada persona usuaria: único e intransferible. Se elaboraría a partir de información de identidad personal e información relacionada con la experiencia particular de violencia de cada persona usuaria, introducida en la App de manera combinada, manualmente por la persona usuaria al responder cuestionarios especializados para la determinación de los niveles de abuso ya existentes, ${ }^{16}$ la $A p p$ identificaría ciertos aspectos de violencia al analizar las comunicaciones y los metadatos que genera el dispositivo móvil en el cual se descarga la aplicación.

El expediente personal puede imaginarse como un receptáculo virtual donde se registrarán todas las comunicaciones de la App relacionadas con la violencia de género, que entran y salen de la aplicación en forma de chat, video, o texto y que podrían tener un valor de apoyo para la generación de historiales de violencia que puedan servir de soporte en los procesos penales. De esta manera el Expediente personal se configura como un elemento esencial para la administración de evidencias.

Los conceptos tutelados en la elaboración y aprovechamiento de este expediente se llaman "privacidad" y "no revictimización". La primera para que a través de las capacidades analíticas del modelo se filtre todo alrededor del expediente y lo que con él se relaciona y la segunda para no redundar con la necesidad de introducir información ya existente; ambos conceptos son particularmente útiles y necesarios para una respuesta adecuada de la policía cuando se pide auxilio y para generar el historial de violencia que puede servir de apoyo al presentar una denuncia ante la autoridad ministerial.

\section{Alertamiento dinámico}

El Alertamiento dinámico es un concepto del modelo de intervención que operaría a través de App para medir los niveles de riesgo, reales y potenciales, de los tipos de abuso a que ha sido expuesta la persona usuaria y que tienen una relación más directa con los hechos de violencia extrema, como es el caso de los feminicidios perpetrados por la pareja íntima y la cadena de hechos relacionados en el continuo de la violencia, como por ejemplo golpizas, violaciones, torturas, entre otros. Incluye la posibilidad de emisión de alertas para las personas en riesgo y para los actores locales según corresponda el nivel y tipo de riesgo que se detecte. El propósito es que las mujeres, adolescentes y niñas usuarias de Tārah 5 cuenten con el apoyo de los actores involucrados en los niveles inferiores de violencia sin que deje de actuar el modelo cuando exista mayor urgencia de protección de las vidas de la persona usuaria, lo que hace conectando a la persona usuaria con la policía, por ejemplo.

16 Los cuestionarios y algoritmos necesarios para la generación de los niveles de riesgo se definirán a partir de las consultas que se hagan con los actores locales existentes y de su experiencia y conocimiento para determinar los niveles de violencia local. 
Adicionalmente y producto de la capacidad de análisis predictivo considerado por el modelo, se ha pensado en incluir la emisión de alertas para recomendar a la persona usuaria conforme se desplaza por la localidad donde se implementa, que evite distintas áreas o puntos de la ciudad donde se conoce que existe una alta probabilidad de ser víctimas de distintos hechos de violencia de género, particularmente cuando, resultado del análisis predictivo, se encuentren coincidencias entre los perfiles de las víctimas de los hechos históricos documentados, con las características de la persona usuaria contenidas en el expediente personal del modelo Tārah 5. Esto es factible alimentando el modelo con bases de datos disponibles sobre hechos delictivos directamente relacionados con la violencia de género y georreferenciados con propósitos preventivos.

\section{Administración de la evidencia}

El modelo de intervención Tārah 5 contempla equipar a la App con la capacidad para administrar evidencias de forma que apoye a las víctimas de abuso en su búsqueda de justicia cumpliendo con los estándares legales aplicables. A partir de la capacidad analítica y de almacenamiento de datos, se pueden guardas indicios y pruebas, particularmente argumentativas y de narrativa, y todo tipo de comunicaciones (correos electrónicos, mensajes de texto y de otras aplicaciones como Whatsapp ${ }^{\circledR}$ y Messenger ${ }^{\circledR}$, video, fotografías y mensajes o notas de voz) que sean pertinentes para soportar una denuncia de violencia de género y que hayan pasado por el dispositivo de telefonía celular donde se haya descargado la App del Modelo Tārah 5.

Al ordenar y de cierta manera administrar toda esta evidencia, el modelo Tārah 5 busca un doble objetivo: cerrar los espacios al anonimato en los que generalmente se mueven y se esconden los perpetradores de abusos y de control, y evitar la revictimización de la persona usuaria al evitarle volver a recrear los abusos de que ha sido víctima para presentar una denuncia de hechos. La App del modelo Tārah 5 tendría la capacidad de generar historiales y líneas de tiempo en formatos específicos que incluyeran todo el material almacenado como se menciona arriba, con fotografías, mensajes o notas de voz y de texto, ordenados para presentarse como parte de una denuncia.

La manera de lograr esto es alimentando a la App con los algoritmos que se utilizan en programas de software para uso de policías y fiscalías. ${ }^{17}$

\section{Botón de pánico}

El Botón de pánico es el componente del modelo Tārah 5 que busca atender los casos de mayor urgencia en cuanto a los niveles más altos de violencia directa. Está pensado para que con un simple click en el dispositivo donde se descargó

$\overline{17}$ Existen distintos softwares en el mercado dedicados a la administración de la evidencia de los cuales se puede echar mano para incluir tales capacidades en la App Tārah 5, tales como Liberty Recording, ACISS Systems, Altia-ABM, ARQvault, Axon Evidence, Capture 911, Case Works, Digital Evidence Manager, DigitalOnQ, entre muchos otros. 
el modelo, conecte en tiempo real a la persona usuaria con el centro de control local de la policía. La idea detrás de esto es reducir al máximo los tiempos de respuesta de la policía y asegurar que la persona usuaria tendrá el tipo de ayuda que corresponde a la emergencia de género que se le presenta. Esto significa que el dispositivo móvil transmitirá en tiempo real un "paquete de datos" preparado exprofeso con la información que la policía requiere para atender un caso con perspectiva de género, lo que incluye, además de los datos de identidad de la persona que solicita la ayuda, y de la ubicación geográfica, el historial de abusos y el nivel actual de riesgo en que se encuentra, especificando el tipo de violencia del cual está siendo víctima. El dispositivo se mantendría "enganchado" con el centro de control de la policía a partir de que este último recibiera la alerta.

Adicionalmente y de manera simultánea, información condensada del evento sería enviada además en tiempo real, al actor que en el modelo Tārah 5 se denomina "Aliado verificador", quien podría, como su nombre lo indica, verificar los tiempos de respuesta y los modos de atención de la policía en cada uno de los casos en que se pidió la ayuda a través del modelo Tārah 5.

Cuando la necesidad de auxilio no amerite llamar a la policía, el Botón de pánico podría ofrecer la opción para solicitar ayuda a un grupo de amigos o familiares en la modalidad de "Acompañamiento seguro".

\section{Acompañamiento seguro}

El Acompañamiento seguro se refiere a la capacidad del modelo para que las personas usuarias puedan mantener contacto a través del dispositivo móvil con un grupo de personas designado por ella misma en la opción "Círculo de confianza", a quienes selecciona y da permisos para que conozcan, en tiempo real y de manera visual, su ubicación y trayectoria geográfica en todo momento durante traslados específicos y que en el modelo Tārah 5 se denomina "Ruta amiga".

Con el acompañamiento seguro, las personas usuarias podrían mantener comunicación con el grupo o con uno de sus miembros a través del chat o de mensajes de voz; igualmente podría enviar video o fotografías de una manera muy similar al uso de Whatsapp. ${ }^{18}$

La Ruta Amiga es la capacidad para trazar una ruta de traslado en la localidad, ya sea en vehículo o a pie, escogiendo, con la ayuda de la capacidad de análisis predictivo del modelo, la ruta que ofrezca las menores probabilidades de ser víctima de un hecho de violencia. La persona usuaria también podría compartir esta ruta con las personas dentro de su "Círculo de confianza", quienes podrían darle seguimiento en tiempo real a través de una interfase en página web.

Tales capacidades serían posibles al alimentar el modelo con las bases de datos históricas de hechos violentos en la localidad, como se explicó en el apartado de "Alertamiento dinámico".

18 WhatsApp Messenger (o simplemente WhatsApp) es una App de mensajería instantánea para teléfonos inteligentes, a través de la cual se envían y reciben mensajes, imágenes, videos, audios, grabaciones de audio (notas de voz), documentos, ubicaciones, contactos, gifs, así como llamadas y videollamadas con varios participantes a la vez, entre otras funciones. 
Los autores consideran la necesidad de incluir la capacidad de educar para erradicar las actitudes machistas que pocas veces se ve que tienen relación directa con los feminicidios, porque si bien corresponden a los niveles más bajos de violencia, es de donde se alimentan las acciones de violencia más extrema. Por ello el modelo Tārah 5 ofrece a todas las personas usuarias la posibilidad de reportar cualquier incidente de acoso o de violencia de género. El modelo georreferencia el hecho con la finalidad de visibilizarlo y evitar que se propaguen acciones que comúnmente no se ven como parte del continuo de violencia, como son los piropos, el bullying, los tocamientos, los insultos, entre otros. Los reportes serían de carácter anónimo y contendrían información y narrativas útiles para dimensionar el acoso y la agresión en la localidad donde se implementa el modelo Tārah 5.

El Botón de reporte contribuiría a debilitar los estereotipos que normalizan el acoso y a difundir el argumento de que no hay inocencia en la comisión de faltas o delitos de graves consecuencias. De manera práctica el uso del Botón ofrecería una serie de opciones predefinidas de acoso y permitiría incluir explicaciones sobre el caso que se reporta. Al momento de "enviar" el reporte, el dispositivo móvil georreferenciaría el hecho y lo relacionaría con el tipo de acoso seleccionado e incluiría la información introducida por la persona usuaria, muy similar a las funciones de la aplicación egipcia HARASSmap. ${ }^{19}$

\section{Muro de experiencias}

El Muro de experiencias está pensado como un blog dedicado como parte del modelo Tārah 5, para permitir la comunicación en grupo y a las personas usuarias comunicar y expresarse libremente sobre sus experiencias de violencia de género. Se ha contemplado que pudiera tener además un espacio privado que funcionara a manera de "diario secreto" de las personas usuarias donde pudieran escribir e incluir fotografías, videos, mensajes o notas de voz.

Se contempla que el espacio público del Muro incluya un espacio abierto para la compartición de información entre el universo de personas usuarias del modelo Tārah 5. Este espacio estaría dedicado a la libre expresión sobre el tema de la violencia contra las mujeres, las adolescentes y las niñas con capacidades muy similares a las que ofrece Twitter ${ }^{20}$ con la posibilidad de "seguir" a otras personas usuarias y de recibir alertas sobre publicaciones particulares.

19 HARASSmap una iniciativa voluntaria que tiene por objeto poner fin a la aceptación social del acoso y la agresión sexual en Egipto. Cuando alguien experimenta o es testigo de un incidente de acoso sexual, puede llenar un reporte en línea o enviarlo por SMS, correo electrónico, Twitter o Facebook, incluyendo los detalles del incidente, así como la dirección, el nombre de la calle y los puntos de interés público. HARASSmap verifica entonces las denuncias y las coloca en un mapa de Google de Egipto, que localiza los puntos de interés de acoso sexual. El mapa muestra puntos rojos donde se han producido incidentes de acoso sexual y de esa manera, sociedad y gobierno pueden actuar con distintas iniciativas orientadas a combatir tales comportamientos y hechos. HARASSmap. https://harassmap.org/en/

20 Twitter es un servicio estadounidense de microblogging y de redes sociales en el que los usuarios publican e interactúan con mensajes conocidos como "tweets". Los usuarios registrados pueden publicar, expresar su 
El Muro de experiencias apuesta a lograr convertirse en un colaborador para los esfuerzos de difusión para combatir la violencia contra las mujeres, las adolescentes y las niñas y conocer las particularidades locales del problema; y para ayudar a que exista una mejor comprensión de las dinámicas de la comunicación entre personas que sufren la violencia en carne propia y ayudar a derrotar "normalidades" de las conductas machistas.

\section{Implementación del modelo TÄRAH 5}

Como en todo proyecto, gran parte del éxito recae en contar con un liderazgo fuerte en términos éticos, funcionales y de autoridad moral. Para lograr una participación comprometida y responsable de grupos de sociedad y gobierno, el modelo Tārah 5 necesita un liderazgo imparcial para su implementación, capaz de convocar a todos los actores necesarios para atender lo urgente y lo importante; capaz de organizarlos alrededor de un objetivo superior, que es combatir juntos el problema de la violencia contra las mujeres, las adolescentes y las niñas a nivel local. Tal liderazgo tendría la tarea de llevar a cabo el proceso de implementación y se convertiría en un testigo de calidad y en consultor estratégico una vez puesta en marcha la operación del modelo Tārah 5 (Ilustración 3).

Por esas razones se ha pensado en la conveniencia de que el modelo Tārah 5 sea propuesto por un organismo internacional o regional con suficiente autoridad moral e imparcialidad, como ONU Mujeres, Igualdad de género y desarrollo (Banco Mundial) y la Comisión Interamericana de Mujeres, entre otras. El trabajo de tal liderazgo sería el de preparar el terreno para la implementación del modelo Tārah 5 que podría lograrse en los siguientes pasos:

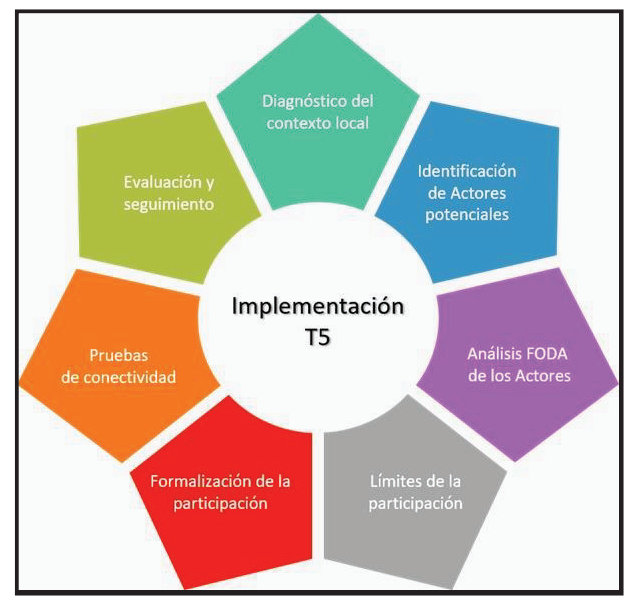

Ilustración 3. Proceso de implementación de Tārah 5. Fuente: elaboración propia.

agrado y volver a twittear tweets, pero los usuarios no registrados sólo pueden leerlos. Los usuarios acceden a Twitter a través de la interfaz de su sitio web, por medio del servicio de mensajes cortos (SMS) o de la App para dispositivos móviles. 


\section{Diagnóstico del contexto local}

A través de este diagnóstico se dimensionaría el problema de la violencia contra las mujeres, las adolescentes y las niñas a nivel local identificando los aspectos de atención urgente y aquellos de mayor importancia para interrumpir el continuo de la violencia, los esfuerzos y estructuras e infraestructuras existentes para la atención de la violencia contra las mujeres, las adolescentes y las niñas y la operación de la aplicación Tārah 5, como es el despliegue de la policía con capacitación para la atención de la violencia de género, la ubicación de refugios para mujeres, las bases de datos sobre la violencia contra las mujeres, las adolescentes y las niñas, un censo de agrupaciones u organizaciones que trabajen en distintos aspectos para atender la violencia contra las mujeres, las adolescentes y las niñas y que serían los potenciales actores para trabajar a través de Tārah 5, los mapas de cobertura de la red de telefonía celular y de wifi público, la ubicación de los servicios de emergencia, entre otros.

\section{Identificación de actores potenciales}

Una vez identificados los grupos de sociedad y gobierno susceptibles a ser parte del esfuerzo Tārah 5, se inicia la selección de los actores existentes según sus especialidades y capacidades de acuerdo con las prioridades de atención definidas en el diagnóstico. A partir de ahí se seleccionaría un pool de candidatos potenciales a quienes se entrevistaría para conocer y comprobar sus capacidades reales de atención de la violencia contra las mujeres, las adolescentes y las niñas.

\section{Análisis FODA de los actores}

Será necesario realizar un análisis de fortalezas y debilidades FODA para cada uno de los actores potenciales con el fin de decidir su participación. Es importante notar que además de sus capacidades de atención de la violencia contra las mujeres, las adolescentes y las niñas, una de las características importantes que deben tener estos actores es su voluntad y compromiso manifiesto de articularse con el resto de los actores a través del modelo Tārah 5 para combatir la violencia contra las mujeres, las adolescentes y las niñas como un todo. A partir de este ejercicio se hace la selección buscando una participación balanceada según las especialidades de cada uno y de acuerdo con el tablero de acción de Tārah 5.

\section{Limites de la participación}

Esta es una etapa de consultas y negociaciones donde se definiría la participación de cada uno de los actores seleccionados y se transparentarían sus capacidades y limitaciones. Sería el momento para reconocer los límites jurídicos y operativos que impone el contexto local para la implementación del modelo Tārah 5 , como es el almacenamiento, resguardo y transmisión de datos personales y la información contenida en el paquete de datos del Botón de pánico, la participación 
de la policía, entre otras; y a partir de ello se definirían los alcances de la implementación del modelo y el periodo de tiempo que se mantendría en operación. Dicho de otra forma, en esta etapa se definiría qué se puede esperar y qué no se debe con la implementación del modelo y de las capacidades de su componente tecnológico, y de cada uno de los actores participantes.

\section{Formalización de la participación}

Será necesario dejar por sentado el compromiso de la participación de todos los actores involucrados con el fin de crear certidumbre entre los participantes, actores y personas usuarias, y de cohesión entre el grupo. De esta manera se podrían definir las capacidades y los materiales que cada actor pondría a disposición de las personas usuarias a través del modelo Tārah 5 y los parámetros para la generación de niveles de riesgo de violencia, los criterios para la emisión de alertas y sus características, mismos que servirían como guías para la elaboración de los algoritmos que conforman la capacidad analítica del componente tecnológico Tārah 5. Esta etapa de consultas culminaría con la formalización de acuerdos de participación y la elaboración de los correspondientes manuales de operación para cada actor de manera individual.

\section{Conclusión}

La violencia contra las mujeres, las adolescentes y las niñas está profundamente arraigada en la sociedad y por esa razón es muy difícil atacar los aspectos ocultos en las estructuras sociales y en nuestra cultura; porque es ahí donde se originan de manera silenciosa e invisible, la violencia directa, física y visible. Empero, el modelo Tārah 5 toma en cuenta la complejidad del problema y toma conciencia de la urgente necesidad de atenderlo porque es un fenómeno que va en aumento. Por ello los autores del modelo creemos que un enfoque de seguridad, centrado en una protección específica, a través de los componentes explicados ofrece una posibilidad de intervenir para atender simultáneamente en lo que es urgente y en lo que es importante.

Cierto es que el camino para resolver el problema es largo, pero el modelo puede verse como una herramienta de contención para abrir espacios desde donde se puedan lanzar políticas públicas y legislación para fortalecer la tendencia que con seguridad estimulará la implementación del modelo Tārah 5, ya que este ofrece provocar cambios de manera tangible en universos masivos de personas. Lo que queda claro es que, conociendo la complejidad del problema, como sociedad no debemos esperar que las políticas y leyes que se concentran en la atención de aspectos parciales del problema lo vayan a resolver; tampoco podemos esperar cruzados de brazos mientras siguen aumentando los feminicidios y los abusos contra las mujeres, las adolescentes y las niñas en todos los puntos del continuo de la violencia. Debemos permitirnos intentar modelos que, operando en la práctica conceptos elementales como los que ofrece el modelo Tārah 5, abarquen todo el espectro de la violencia contra las mujeres, las adolescentes y las niñas como 
un todo; de continuar con esfuerzos aislados, seguiremos siendo testigos de asesinatos de mujeres donde las víctimas pidieron ayuda numerosas veces a distintos actores sin que se articulara la ayuda y la protección necesarias. Por citar un ejemplo, el 25 de abril de 2020, Vanesa Gaytán Ochoa de 25 años fue acuchillada en varias ocasiones por su pareja sentimental frente a la residencia oficial del gobernador de Jalisco. Antes de ese hecho que le causó la muerte frente a los policías que resguardaban la residencia, Vanesa buscó ayuda en 16 ocasiones al Centro de Justicia para las Mujeres, pero no se dio seguimiento a su caso y no se actuó para protegerla (Cuarto Poder, 2020).

El modelo que aquí se presenta es una idea sustentada en sólidas teorías de feministas y estudiosos de la violencia, que ha sido viabilizada a través de la experiencia de sus autores en el campo de la práctica, por lo que puede ser parte de una estrategia de intervención para contener y prevenir las manifestaciones más extremas de la violencia contra las mujeres, las adolescentes y las niñas.

El uso de la tecnología de las comunicaciones y del aprendizaje automático (machine learning) ofrece oportunidades como nunca para conectar, coordinar, sensibilizar y proteger a universos masivos de personas. La importancia y urgencia de encontrar soluciones a un problema que ha enfermado a nuestra sociedad nos obliga a echar mano de todos los recursos que estén a la mano y no hay un solo aspecto tecnológico incluido en modelo Tārah 5 que no exista ya en el mercado o que no se pueda adquirir.

No hay que olvidar que el Estado tiene una obligación formal de articular y desarrollar acciones para combatir a la violencia contra las mujeres, las adolescentes y las niñas de manera transparente, y la sociedad una obligación moral para resolver un problema que desconfigura nuestras comunidades y envenena nuestra convivencia. "El problema es que una vez que lo ves, no puedes 'des-verlo'. Y una vez que lo has visto, callar, no decir nada, se convierte en un acto tan político como hablar. No hay inocencia. De cualquier manera, eres responsable" (Arundhati Roy).

\section{Bibliografía}

Butler, Judith, Gender trouble: Feminism and the subversion of identity, New York, NY, Routledge, 1993.

Cockburn, Cynthia, "The Continuum of Violence: A Gender Perspective on War and Peace", Wenona Giles, Sites of Violence: Gender and Conflict Zones, California Scholarship Online: March 2012, https://doi.org/10.1525/california/9780520230729.001.0001.

Cuarto Poder, "Mujer asesinada pidió auxilio 16 veces". https://www.cuartopoder. $\mathrm{mx} /$ nacional/mujer-asesinada-pidio-auxilio-16-veces/311067/.

Declaración sobre la eliminación de la violencia contra la mujer, proclamada por la Asamblea General en su resolución 48/104 de 20 de diciembre de 1993, https://www.ohchr.org/EN/ProfessionalInterest/Pages/ViolenceAgainstWomen.aspx 
Galtung, Johan, Violence, Peace, and Peace Research, Journal of Peace Research, vol. 6, núm. 3 (1969), pp. 167-191, Published by: Sage Publications, Ltd.Stable. http://www.jstor.org/stable/422690.

Gould, Laurie A., "Exploring the Relationship Between Gender Violence and State Failure: a CrossNational Comparison", Georgia Southern University, Statesboro, USA, Violence Against Women 2016, vol. 22(11), 1343-1370, p. 1344, sagepub.com/journalsPermissions.nav, https://doi.org/10.1177/1077 801215624790.

Gray, Harriet and Cockburn, Cynthia, "Anti-Militarism: Political and Gender Dynamics of Peace Movements", International Feminist Journal of Politics, 16:1, 168-170, https://doi.org/10.1080/14616742.2013.876293, 2014, https:// doi.org/10.1080/14616742.2013.876293.

Henkeman, Sarah et al, Transdisciplinary project for social justice, Open Guide to a Deeper, Wider and Longer Analysis of Violence, 2016. https://www.researchgate.net/publication/312587585_Open_Guide_to_a_Deeper_Wider_ and_Longer'_analysis_of_violence.

Hughes, Melany, "The intersection of gender and minority status in national legislatures: The minority women legislative index", Legislative Studies Quarterly, XXXVIII, 4, November 2013, 489, https://doi.org/10.1111/1sq.12025. JAYAKUMAR, Kirthi, "Redrawing the Galtung Triangle-Finding Place for Healing Trauma", Peace Work, 17 Jun 2019. https://www.transcend.org/tms/2019/06/ redrawing-the-galtung-triangle-finding-place-for-healing-trauma-in-peacework/.

KINDERVAG, John, Build Security into your network's DNA: The Zero Trust Network Architecture, Forrester Research, Inc., 2010. http://www.virtualstarmedia. com/downloads/Forrester_zero_trust_DNA.pdf

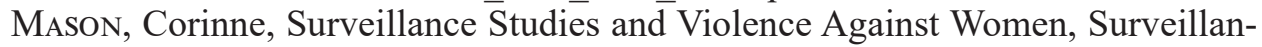
ce \& Society, University of Ottawa, Canada, 2012.

Moser, Caroline, The gendered continuum of violence and conflict: An operational framework, 2001. In: Caroline Moser and Fiona Clark (eds), Victims, Perpetrators or Actors? Gender, Armed Conflict and Political Violence. London: Zed Books. In: A holistic approach to violence: Women parliamentarians' understanding of violence against women and violence in the Kurdish issue in Turkey, p. 78, European Journal of women's Studies 2016, vol. 23(1) 76-92, 201. sagepub.co.uk/journalsPermissions.nav, https://doi. org/10.1177/1350506814554487.

SchePer-Hughes, Nancy, Death Without Weeping: The Violence of Everyday Life in Brazil, University of California Press, 1992, p. 614.

Sentencia de la Suprema Corte de Justicia de la Nación, Caso Mariana Lima Buendía, 2015.

Spotlight Initiative to eliminate violence against women and girls, Terms of Reference 2017-2023, UN \& EU, 2017.

The World's Women 2015, Trends and Statistics, Chapter 6, Violence against Women, United Nations Department of Economic and Social Affairs, 2015 
and UN Women Global Database on Violence against Women. https://unstats. un.org/unsd/gender/ downloads/Ch6_VaW_info.pdf

United Nations Sustainable Development Goal 5 Achieve gender equality and empower all women and girls. https://sustainabledevelopment.un.org/sdg5. Wood, Julia T., On the Eve of Women's Studies in Communication's 40 Year Anniversary, The University of North Carolina at Chapel Hill, 2014. 